\title{
TRK Receptor Family Alteration Positive
}

National Cancer Institute

\section{Source}

National Cancer Institute. TRK Receptor Family Alteration Positive. NCI Thesaurus. Code C138155.

Indicates that mutation, overexpression, or rearrang ement of an TRK receptor family member has been detected in a sample. 